\title{
Nutrition Challenges of Patients with Alzheimer's Disease and Related Dementias: A Qualitative Study from the Perspective of Caretakers in a Mental National Referral Hospital
}

\author{
Edwin Kigozi $\mathbb{D}^{\prime}$ \\ Clement Egwela' \\ Livingstone Kamoga (D) \\ Scovia Nalugo Mbalinda' \\ Mark Kaddumukasa ${ }^{2}$ \\ 'Department of Nursing, School of \\ Health Sciences, College of Health \\ Sciences, Makerere University, Kampala, \\ Uganda; ${ }^{2}$ School of Medicine, College of \\ Health Sciences, Makerere University, \\ Kampala, Uganda
}

Purpose: The burden of Alzheimer's dementia greatly impacts patients and their immediate families. Studies on the perspective of caretakers regarding nutrition in patients with Alzheimer's disease (AD) dementia are lacking. Yet this information is needed to guide clinical care for patients with dementia. The study explored caretakers' perspective on nutritional challenges faced by patients with Alzheimer's disease and related Dementias at Butabika National Referral Hospital.

Methods: We conducted 20 in-depth interviews and 2 focus group discussions with 20 health workers and 16 caregivers, respectively. The focus group discussions and in-depth interviews were audio-recorded and transcribed. Analysis was conducted using a thematic, constant comparative approach with an emphasis on dominant themes.

Results: Participants had a mean age of 37 in the range (27-44) years. Seventeen (47\%) of them were males. Their duration of Care for Dementia to patients was in the range (2-7) years. The highest level of education was a bachelor's degree and the primary level was the lowest. Thirteen $(35 \%)$ were married and twenty-three $(65 \%)$ were not, and they either survived on salaries or wages as a source of income. The key emerging issues were 1) hindrances to nutritional care in dementia, 2). Factors leading to inadequate nutrition among people with dementia and 3). Recommendations to improve nutrition needs.

Conclusion: Caretakers experience challenges ranging from psychotic manifestations of a patient to hindrances in the provision of nutritional care. A better understanding of their experience is essential for the development of interventions to help the family members, health workers and other care takers promote good nutrition in patients with Alzheimer's dementia. A clear referral system should be established to prevent overcrowding of patients at a mental national referral hospital to ensure adequate timely nutritional support to those admitted. Capacity building programs should continue to address the knowledge gap in nutritional requirements of patients with Alzheimer's dementia.

Keywords: Alzheimer's disease, dementia, nutrition, challenges

\section{Introduction}

Globally, around 50 million people have dementia, with about $60 \%$ living in low- and middle-income countries, with nearly 10 million new cases yearly. A total of 82 million in 2030 and 152 million in 2050 dementia cases are projected due to the rising numbers of people with dementia living in low- and middle-income countries. ${ }^{1}$ Patients with
Department of Nursing, School of Health Sciences, College of Health Sciences, Makerere University, P.O. Box 7072, Kampala, Uganda

Tel +256 75I4I0637; +256 778297240

Email kigoziedwin165@gmail.com 
dementia have nutrition challenges stemming from psychotic manifestations hindering them to be addressed. The caretakers refer to health workers, social workers and family members who offer support to patients.

Dementia is the loss of previous cognitive, executive, and memory function levels in a state of full alertness. It is classified as primary or secondary. Primary dementia is where dementia itself is the major sign of some organic brain disease, is not directly related to any other organic illness and those caused by/related to another disease. ${ }^{2}$ A decline in nutritional status positively correlates with a decline in cognition and vice versa. If nutritional challenges are not addressed, this can increase the rate of deterioration and increasing clinical vulnerability, eg, risk of falls, infections, and pressure sores. ${ }^{3}$

Epidemiologic data suggests that nutritional intake may influence AD's development and progression. ${ }^{4}$ Evidence related to the effect of diet and nutritional factors on the risk of $\mathrm{AD}$ and cognitive aging points to a protective role for certain nutrients, such as omega-3 fatty acids, beta-carotenes, antioxidants like vitamins $\mathrm{C}$ and $\mathrm{E}$ as well as $\mathrm{B}$ complex vitamins whose lack results in hyperhomocysteinemia, a risk factor for atherosclerosis, as well as nutrition-related disorders like hypercholesterolemia, hypertension, and diabetes, recently associated with cognitive impairment in old age. ${ }^{5,6}$

Much as the caregiving burden has been studied extensively in high-income countries, there is a paucity of information about care givers' experiences in low-income countries particularly rural areas of Africa, where health systems for the management of people with Alzheimer's Dementia have not been developed. ${ }^{7}$ Maintaining an individual's nutrition status, including preventing unintentional weight loss, fluid and micronutrients is important in dementia. Since patients with Alzheimer's dementia receive care in both homes and hospitals, those who rely on family support to maintain adequate nutrition have an increased risk of malnutrition. There is limited literature exploring how nutritional care for people living with dementia at home could be improved and the role that care providers have to play. ${ }^{3}$ This study therefore seeks to explore the caretakers' perspective on nutritional challenges faced by patients with Alzheimer's disease and related Dementias as well as providing possible strategies to overcome these challenges.

\section{Materials and Methods Study Design}

This was a cross-sectional qualitative study was conducted using Focus Group Discussions (FGDs) and In-depth Interviews (IDIs) in August and September 2020.

\section{Study Setting}

The study was carried out at Butabika National Referral Hospital (BNRH), the only Mental national referral hospital in Uganda.

\section{Sample Size Estimation, Discussion and Interview Methods}

This study had a total of thirty-six (36) study participants were purposively selected in the study. For qualitative research, this sample size is within the recommended number of 20-50 individuals. ${ }^{8,9}$ Participants of age 18 years or older, cognitively intact, and able to provide informed consent were recruited in the study. Subjects unable to provide written informed consent and those with secondary epilepsy due to traumatic brain injury or stroke were excluded. Two separate Focus Group Discussions (FGDs) were conducted with each consisting of 8 caretakers, who were a mixture of social workers and patients' family members. An experienced qualitative researcher fluent in both English and Luganda (local language of the study area) moderated the FGDs. The FGDs were conducted to give in-depth insights into the nutrition issues of people with dementia. We conducted in-depth interviews in 20 health-care providers. Both in-depth interviews and FGDs were conducted within the hospital compound.

\section{Qualitative Data Collection and Analysis}

Using both face-to-face in-depth interviews and FDGs, narrative data on nutrition in dementia was utilized to optimize credibility and validity of this study. ${ }^{10,11}$ We used openended questions so as not to limit the range or breadth of discussion among the participants. We positioned the study participants for adequate eye contact with others in the group and the discussion lasted approximately one hour.

Some of the examples of the open-ended questions used to explore the nutrition issues included; "What challenges do you experience while providing good nutrition for your patient?" The guide also included examples of follow-up probes such as "would you explain further", "please describe what you mean", and "would you give me an example". The interview was recorded, and then transcribed verbatim. Information collected from focus group sessions included interview and observation. A team of three investigators (EK, LK and CE) read the focus group transcripts in their entirety to gain familiarity with the data. Segments of text from the transcript were labeled and assigned codes that described meaning of content. ${ }^{9}$ The codes were subsequently collapsed into 
broad themes or categories. EK, LK and CE independently coded each transcript to ensure consistency and transparency of the coding; discrepancies were resolved by discussion. Finalization of codes was based on the consensus of the qualitative team. We utilized the grounded theory approach to data analysis, involving open, axial and sequential coding, and the constant comparative method to generate constructs (themes) and elaborate the relationship among them. ${ }^{12}$ A separate coding dictionary was then constructed for the interviews and focus groups.

\section{Results}

\section{Participants' Characteristics}

The mean age of all participants was 37 years (27-44) and $17(47 \%)$ of study participants were males. The duration of care for Dementia by participants was in the range of two (2) to seven (7) years with respect to time of diagnosis. The highest level of education of caretakers was a bachelor's degree while the lowest level of education was primary level. Of the caretakers, 13 (35\%) were married and 23 (65\%) were unmarried, who either survived on salaries or wages as a source of income.

Three different themes were generated from this study which include 1) Hindrances to nutritional care in dementia, 2). Factors leading to inadequate nutrition among people with dementia and 3). Recommendations to improve nutrition needs.

\section{I) Hindrances to Nutritional Care in Dementia}

This included two (2) sub-themes: hindrances due to psychotic manifestations in dementia and those related to other factors.

\section{Hindrances Due to Psychotic Manifestations in Dementia}

Patients with Alzheimer's dementia manifest with psychotic symptoms such as restlessness, aggressiveness, violence, confusion, irritability, aggression, wandering around and disorientation that hinder their nutrition at home or within communities.

The nature of behavior of the patient while at home and in the community tend to bring in signs that are associated with dementia like aggression, irritability, and confusion, hence brought to hospital for medical care. (Respondent 2, Family member
The nature of their illness which makes them move without insight, wandering around hence escaping from the hospital before recovery from their nutritional challenges due.

... ... their illness makes them wander around with no insight, thus escaping from the hospital before recovery. (Respondent 6, Nursing officer)

\section{Hindrances Related to Other Factors}

Caregiver role strain reflected by a high Patient-Caretaker ratio leaves limited time for ensuring hygiene, serving food and supervising patients with dementia, thus hindering effectiveness and efficiency of care given.

... ... right now, I have many patients amidst three (3) staffs on duty, hence challenging to ensure hygiene and serving food and supervising patients so that the energetic ones they don't grab food from the elderly. (Respondent 11, Senior Nursing Officer)

Ageing makes them vulnerable thus require special nutritional care which the hospital cannot provide, for example, they forget to eat, how to hold and use utensils, develop difficulty in communicating desired foods, chewing and swallowing. Hence have to be fed by the caretakers in the hospital to ensure that they eat enough food.

Old people are vulnerable in that they forget to eat, holding and using utensils, find it difficult to communicate the food they want, chew and swallow. You have to be there to see what they have eaten and how much they have eaten. (Respondent 10, Mental Health Nurse)

Multiple ethnicities of patients admitted at the hospital results in different food preferences where some patients prefer their local traditional foods rather than what is provided. This fixation to their staple food leads to a decrease in appetite ending up refusing to eat the food provided.

We have different tribes with different food preferences, yet here we have only posho and beans. Someone wants 'kaloo' (millet bread), sometimes they refuse to eat. Acutely disturbed patients have no time to eat at times, they even pour away the food. Some also have poor appetite. (Respondent 5, Psychiatric Nurse)

\section{2). Factors Leading to Inadequate Nutrition Among People with Dementia}

Several factors were reported responsible for inadequate nutrition among patients with Alzheimer's dementia 
including knowledge, financial and caregiver support factors, forming the different sub-themes.

\section{Knowledge Factors}

Caregivers reported lack of knowledge to improve and encourage better eating-drinking habits in patients with Alzheimer's dementia so as to maintain good nutrition.

I really don't know how frequent I should give food and drinks to my mother. Also, I can't tell which portions of foods, drinks, and snacks my mother should be given on a daily basis. (Respondent 17, Family member)

Lack of Continuous Mental Health Education (CME) sessions on nutrition in dementia leave a gap in knowledge among health workers concerning nutrition among patients with Alzheimer's dementia.

We had one CME on nutrition in which little knowledge was shared pertaining nutrition in dementia (Respondent 9, Mental Health Nurse).

Lack of health education on nutritional needs of patients with Alzheimer's dementia by health workers upon discharge leaves home caretakers with limited knowledge and awareness pertaining patients' nutrition.

....... we are not taught about our patients' nutrition here at hospital before discharging them, this leaves us challenged not knowing what to do ...... (Respondent 5, Family member).

Caretakers mainly from the hospital setting have inefficiency in capacity building to improve on their quality-ofservice delivery. The salary offered to them is also low for them, thus requires to be revised and increased.

Capacity building is not as efficient as it is meant to be so as to improve the quality-of-service delivery, in addition, the salary offered to them is low hence a need for revision and increase. (Respondent 20, Nursing Officer)

\section{Financial Factors}

Caretakers reported lack of financial support to cater for different kinds of food including diet supplements containing micronutrients such as omega-3 and 6 and vitamins or medication to stimulate appetite in their patients. This predisposes patients to malnutrition.

....... the major challenge is feeding the clients with Alzheimer's dementia on a single diet, that is, posho and beans without a supplement since we lack finances to cater for dietary supplementation. (Respondent 7, Senior Nursing officer)

Furthermore, being a government hospital, its funding is part of the share from national budget and financial support from donations, which is not enough to cater for nutritional needs of patients with Alzheimer's dementia at the hospital.

...... the funding from the national budget is not enough, and so is the money from donations, hence lack of finances remains a major hindrance in provision of better nutrition to patients we admit. .... (Respondent 3, Nursing Officer)

\section{Caregiver Support Factors}

Some patients are abandoned by their relatives and left for the hospital to satisfy their nutritional demands. This is because family members lack time to take care of their patients within the home environment even when resources are available.

..... we bring them to the hospital because we have no time to care for them at home, irrespective of whether we have resources or not, we therefore we abandon them here ......... (Respondent 4, Family member)

Due to diverse responsibilities and the busy schedule of the family members in homes in form of household chores, farm work and other duties, patients end up missing some daily meals like breakfast and lunch, which is another challenge.

....... at home, they can fail to get their meals such as breakfast and lunch since we wake up early in the morning going to our gardens for work and they are left home waiting ........ (Respondent 7, Family member)

\section{3). Recommendations to Improve Nutritional Needs}

Health-care workers viewed it important for each of them and other caretakers to be knowledgeable on nutrition management of patients with Alzheimer's dementia. This could be through various trainings offered by the hospital management and other responsible bodies. The hospital should send its health workers for refresher courses and also offer facilitation to them.

Every health worker should undergo the training in nutrition management of clients with Alzheimer's dementia. (Respondent 15, Psychiatric clinical officer). 
Through use of the media such as television, radio talk shows and newspapers, increase in caretakers' knowledge on nutrition among patients with Alzheimer's dementia can be achieved.

... Putting more funds for training health workers in nutrition education is good but there should also be talk shows on radios, televisions and columns in the newspapers about nutrition in dementia this could help the relatives too. (Respondent 13, Nursing Officer)

The hospital management should mind about improving the diet of the Alzheimer's dementia patients through addition of certain cheap foods with more nutritive values like grains, nuts, fruits, milk, seafood/silver fish and vegetables. Their focus should not only be on expensive foods like chicken and beef.

... ... ... should improve on the diet by supplementing with ground nuts, milk, bananas, 'mukene' (silver fish), and greens not only thinking of meat or 'nkoko' (chicken). (Respondent 18, Nursing Officer)

Referral system should be followed to limit the number of patients in the hospital for effective and efficient service delivery. Due to the fact that the ratio of nurses to patients is low, and the ratio of wards to patients is low too, there is a lag in service delivery, hence the need to decongest the hospital by following the referral system of patients to other hospitals.

To me the referral system of admissions should be followed to decongest the hospital for other regional referral hospitals to handle some patients. This will lead to a limited number that can be handled. (Respondent 1, Senior Nursing Officer)

\section{Discussion}

This study looked at the nutritional challenges among patients with Alzheimer's dementia through assessment of their caretakers. Many quantitative studies have looked at different associations between malnutrition and dementia patients' associated factors. ${ }^{5,13-15}$ Under nutrition is particularly common among people with cognitive decline and Alzheimer disease. ${ }^{16}$

In this study, it was noted that people with Alzheimer's dementia exhibit behaviors like aggressiveness, restlessness, confusion, irritability, disorientation, forgetfulness, violence and wandering around, which is attributed to the disease's effect on their neurological functions. A related study conducted in southwestern Uganda showed that participants identified cognitive symptoms such as memory loss as well as behavioral changes associated with dementia where forgetfulness was most reported. ${ }^{17}$ Other studies showed that people with dementia are fond of escaping, wandering, or spoiling things, ${ }^{18}$ forgetting their places of residence and being aggressive. ${ }^{19}$ These similarities in the presentation shown by these studies are as a result of a common cause and progression of the disease condition in all clients.

This study pointed out malnutrition as one of the nutritional challenges patients with Alzheimer's dementia face as a result of protein, carbohydrates, micronutrients such as omega-3 and 6 fatty acids and vitamin deficiencies. A similar study conducted among institutionalized patients with Alzheimer's dementia found that one fourth of the patients had vitamin and flavonoid deficiency, high percentage of patients with plasma deficit of iron and zinc was noteworthy. ${ }^{20}$ The similarity in results obtained can be attributed to the fact that there is inadequate professional nutritional support from dietitians in both settings. Similarly, malnutrition occurs frequently among elderly individuals. It has been shown that people with Alzheimer's dementia may have more nutritional problems than healthy people. ${ }^{21}$ Therefore, proper nutrition has an impact on the recovery of the patient since nutrition not only influences the individual's health status but it is also important in the maintenance of the immune system function, production of blood elements, and for the synthesis of serum proteins. ${ }^{20}$ There is therefore need to also improve the diet of patients in BNRH since nutritional supplements lower morbidity and mortality. ${ }^{20}$

It was noted that most of the patients need to be supervised and fed during their time of being at the hospital. This is because most of them are very old hence vulnerable and tend to refuse eating what is provided to them without supervision. A related study on nutritional risk factors among patients with Alzheimer's dementia showed that regarding independence in feeding behavior, subjects who were nutritionally okay with a Mini Nutritional Assessment (MNA) score below the median more often had problems feeding themselves. ${ }^{22}$ Therefore, the point of vulnerability due to old age is a reason for the similarity of results in both studies.

The current study revealed that there is ignorance among care takers pertaining proper clients' nutrition in Alzheimer's dementia. This is attributed to the low level of caretaker empowerment through education as far as their 
clients' nutrition is concerned. This is in line with a study carried out in Finland which revealed that the professionals had not understood, before the education, the connection of nutrition to the comprehensive well-being of aged residents. $^{23}$

The study revealed home-associated nutritional challenges where their caretakers rarely have time for them, hence are abandoned by relatives and left in the hospital. Abandonment is as a result of the weird behavior they express, which brings about discrimination of clients with Alzheimer's dementia thus inability to cater for their needs in nutrition such as timing meals therefore end up missing them. In a study conducted in France on assessment of home caretakers, noted that meal timing problems by home care caregivers could cause distress for people with dementia. This underlines the importance of assessing nutritional status in the context of dementia because nutritional deficiencies co-exist with other morbidity and care difficulties and may compromise the quality of life of people with dementia. ${ }^{24}$

It was noted in the current study that there is role strain due to work over-load resulting from a high patient-caregiver ratio thus end up not doing their best. Similarly, it was shown that patients with malnutrition have more behavioral disorders (high Neuropsychiatric Inventory score) and the burden felt by the caretaker seems heavier. ${ }^{14}$ Therefore, work in multi-professional teams is very useful in delivery of services to patients. ${ }^{23}$ Due to the fact that the current study found out few health-care workers attending to the patients with dementia, there is need for recruitment of more health-care workers for effectiveness and efficiency of service deliveries.

The current study showed more nutritive support within the hospital than in the patients' homes of residence. This is due to the presence of more supervision for clients in hospitals than in homes. However, a study done in Finland showed that dementia ward residents suffered from moderate to severe dementia and were at the end stage of the disease than those in homes. Therefore, it can be speculated whether at some point of the terminal stage the demented individuals no longer respond to nutritional intervention. ${ }^{23}$ The difference in the findings is due to the differences in the study area, more especially in terms of nutritional support offered to clients.

The current study showed that caretakers had never attended any education session specifically on the nutrition among patients with dementia. The absence of trainings on nutrition among patients with Alzheimer's dementia in the current study was not the case in a similar study where professionals responded that during the refresher trainings, they learned to evaluate how the patients' meals correspond to the nutritional recommendations given to this age group. ${ }^{23}$ There is therefore need for caretakers in the current study to have trainings on the nutritional needs of patients with Alzheimer's dementia because these nutritional educational programs for home caregivers can reduce the occurrence of nutritional deficiencies to persons with dementia and improve their health.

The current study revealed that caretakers do not receive capacity building trainings as a way to address nutrition needs in patients with Alzheimer's dementia. This is not similar to what a certain study showed that services were provided by health-care workers and ranged from providing information to the patients and caregivers about benefits of a balanced diet in some facilities to the actual preparation and serving of food to patients in other facilities. In addition, some facilities provided food stuffs such as maize flour, sugar, among others to the patients and their families. ${ }^{25}$ The observed difference in service provision depends on the need observed since both studies are carried out from different localities.

\section{Study Limitations}

The small convenient sample utilized in this study, and the conduct of the study in a single urban area in Uganda may limit transferability of the study findings. In addition, nutrition challenges were self-reported and might have been influenced by recall bias. However, these limitations are offset, to some extent, by the utilization of rigorous qualitative methods described in the study and our use of the Consolidated Criteria for Reporting Qualitative research (COREQ) (Tong A, Sainsbury P, Craig J. Consolidated criteria for reporting qualitative research (COREQ): a 32-item checklist for interviews and focus groups. Int J Qual Health Care. 2007;19(6):349-357), to improve the rigor, comprehensiveness and credibility of the interviews and focus groups.

\section{Conclusion}

Care takers of patients with Alzheimer's dementia experience a wide range of challenges surrounding nutritional support of their patients. A better understanding of the care takers experience is essential for the development of strategies to help the family members, health workers and other care takers promote good nutrition in clients with Alzheimer's dementia. Integration and emphasizing nutritional challenges 
for patients with Alzheimer's dementia during routine medical care would help alleviate these challenges.

\section{Abbreviations}

AD, Alzheimer's disease; BNRH, Butabika National Referral Hospital; CME, Continuous Medical Education; CMHE, Continuous Mental Health Education; FGD, Focus Group Discussion; IRB, Institutional Review Board; IDI, In-depth Interview; $\mathrm{MOH}$, Ministry of Health; PI, Principal Investigator; WFP, World Food Program; WHO, World Health Organization.

\section{Ethics Considerations}

Approval to conduct the study was obtained from the Institutional Review Board of TASO, Mulago under Ref: TASOREC/042/2020-UG-REC-009. This study was conducted in accordance with the Declaration of Helsinki, where by, Individual voluntary, written informed consent was sought from the participants for their participation in the study. The protection and confidentiality of their identity, and their right to withdraw from the study at any time was observed. Interviews were recorded with participants' permission. All original documents, including the consent forms, were stored in a secure locked cabinet and digital transcriptions stored in secure password protected files only accessed by the researchers. In addition, the participant informed consent included publication of anonymized responses from the study participants.

\section{Acknowledgments}

We give thanks to God for having enabled us carry out this research study successfully. We acknowledge the support from Ronald Olum, Faith Atai, Emmanuel Balinda, Banadda Patrick Matovu, Kamoga Daniel Wasswa, Robinah Namagembe and Jemba Pius for their contribution during the study time. We acknowledge the management and staff of Butabika National Referral Hospital, and all the research participants for the cooperation and assistance rendered to us during the study time.

\section{Author Contributions}

All authors contributed to conceptualization and design of the study, data analysis, interpretation as well as drafting and revising the article, have agreed on the journal to which the article will be submitted, gave final approval of the version to be published, and agree to be accountable for all aspects of the work.

\section{Funding}

Research reported in this publication was supported by the Fogarty International Center of the National Institutes of Health, US Department of State's Office of the US Global AIDS Coordinator and Health Diplomacy (S/GAC), and President's Emergency Plan for AIDS Relief (PEPFAR) under Award Number 1R25TW011213. The content is solely the responsibility of the authors and does not necessarily represent the official views of the National Institutes of Health.

\section{Disclosure}

The authors report no conflicts of interest in this work.

\section{References}

1. Risk reduction of cognitive decline and dementia: WHO; 2019. Available from: https://www.who.int/publications/i/item/risk-reduc tion-of-cognitive-decline-and-dementia. Accessed April 20, 2020.

2. Townsend MC, Morgan KI, editors. Psychiatric Mental Health Nursing Concepts of Care in Evidence-Based Practice. 9th Edition; Kindle. F.A. Davis Company; 2017.

3. Mole L, Kent B, Abbott R, et al. The nutritional care of people living with dementia at home: a scoping review. Health Soc Care Community. 2017;26(4):e485-e496. doi:10.1111/hsc.12540

4. Swaminathan A, Jicha GA. Nutrition and prevention of Alzheimer's dementia. Front Aging Neurosci. 2014;6:282. doi:10.3389/ fnagi.2014.00282.

5. Salerno-Kennedy R, Cashman KD, Salerno-Kennedy C. Relationship between dementia and nutrition-related factors and disorders: an overview. Int J Vit Nutr Res. 2005;75(2):83-95. doi:10.1024/03009831.75.2.83

6. Otaegui-Arrazola A, Amiano P, Elbusto A, Urdaneta E, MartínezLage P. Diet, cognition, and Alzheimer's disease: food for thought. Eur J Nutr. 2014;53(1):1-23. doi:10.1007/s00394-013-0561-3

7. Ainamani HE, Alele PE, Rukundo GZ, et al. Caring for people with dementia in rural Uganda: qualitative study of caregiving burden experienced by informal and formal caregivers. $J$ Glob Health Rep. 2020;4(10). doi:10.29392/001c. 12848

8. Creswell JW. Qualitative inquiry \& research design: choosing among five approaches; 2007. Available from: https://psycnet.apa.org/record/ 2006-13099-000. Accessed July 16, 2021.

9. Denzin NK, Lincoln YS. The Sage Handbook of Qualitative Research (3rd Edition). London: Sage; 2005.

10. Creswell JW, Miller: DL. Determining validity in qualitative inquiry. Theor Pract. 2000;39(3):4-7. doi:10.1207/s15430421tip3903_2

11. Patton MQ. Enhancing the quality and credibility of qualitative analysis. Health Serv Res. 1999;34:1189-1208.

12. Strauss: AL, Strauss A. Qualitative Analysis for Social Scientists. New York, NY: Cambridge University Press; 1988. doi:10.1017/ CBO9780511557842

13. Faxén-Irving G, Basun H, Cederholm T. Cederholm, Nutritional and cognitive relationships and long-term mortality in patients with various dementia disorders. Age Ageing. 2005;34(2):136-141. doi:10.1093/ageing/afi023

14. Guerin O, Soto ME, Brocker P, et al. Nutritional status assessment during Alzheimer's disease: results after one year (the REAL French Study Group). J Nutr Health Aging. 2005;9:81-84.

15. Murphy JL, Holmes J, Brooks: C. Nutrition and dementia care: developing an evidence-based model for nutritional care in nursing homes. BMC Geriatr. 2017;17(1):55. doi:10.1186/s12877-017-0443-2 
16. Shlisky J, Bloom DE, Beaudreault AR, et al. Nutritional considerations for healthy aging and reduction in age-related chronic disease. Adv Nutr. 2017;8(1):17-26. doi:10.3945/an.116.013474

17. Owokuhaisa J, Rukundo GZ, Wakida E, et al. Community perceptions about dementia in southwestern Uganda. BMC Geriatr. 2020;20 (1):135. doi:10.1186/s12877-020-01543-6

18. Sandman: P-O. Aspects of Institutional Care of Patients with Dementia. Umeå: Umeå universitet; 1986.

19. Górska S, Forsyth K, Maciver: D. Living with dementia: a meta-synthesis of qualitative research on the lived experience. Gerontologist. 2018;58:180-196. doi:10.1093/geront/gnw195

20. Gregorio PG, Diaz SR, Casado JR. Dementia and nutrition. Intervention study in institutionalized patients with Alzheimer disease. J Nutr Health Aging. 2003;7:304-308.

21. Bamford C, Heaven B, May C, et al. Implementing nutrition guidelines for older people in residential care homes: a qualitative study using normalization process theory. Implement Sci. 2012;7(1):106. doi:10.1186/1748-5908-7-106
22. Andrieu S, Reynish W, Nourhashemi F, et al. Nutritional risk factors for institutional placement in Alzheimer's disease after one-year follow-up. J Nutr Health Aging. 2001;5:113-117.

23. Suominen M, Kivisto S, Pitkala K. The effects of nutrition education on professionals' practice and on the nutrition of aged residents in dementia wards. Eur J Clin Nutr. 2007;61(10):1226-1232. doi:10.1038/sj.ejen.1602639

24. Rullier L, Lagarde A, Bouisson J, et al. Nutritional status of community-dwelling older people with dementia: associations with individual and family caregivers' characteristics. Int J Geriatr Psychiatry. 2013;28(6):580-588. doi:10.1002/gps.3862

25. Niyonzima V, Maling S, Chemali: Z Enablers and barriers to utilisation of available formal support by institutionalised individuals with Alzheimer's disease and related dementias in rural South Western Uganda: a qualitative descriptive study; 2019. doi:10.21203/ rs. $2.15396 / \mathrm{v} 1$

\section{Publish your work in this journal}

Neuropsychiatric Disease and Treatment is an international, peerreviewed journal of clinical therapeutics and pharmacology focusing on concise rapid reporting of clinical or pre-clinical studies on a range of neuropsychiatric and neurological disorders. This journal is indexed on PubMed Central, the 'PsycINFO' database and CAS, and is the official journal of The International Neuropsychiatric Association (INA). The manuscript management system is completely online and includes a very quick and fair peer-review system, which is all easy to use. Visit http://www.dovepress.com/testimonials.php to read real quotes from published authors. 